\title{
Ultrasound imaging in neurosurgery: approaches to minimize surgically induced image artefacts for improved resection control
}

\author{
Tormod Selbekk • Asgeir Store Jakola • Ole Solheim • \\ Tonni Franke Johansen • Frank Lindseth • \\ Ingerid Reinertsen • Geirmund Unsgård
}

Received: 28 January 2013 / Accepted: 6 February 2013 /Published online: 5 March 2013

(C) The Author(s) 2013. This article is published with open access at Springerlink.com

\begin{abstract}
Background Intraoperative ultrasound imaging is used in brain tumor surgery to identify tumor remnants. The ultrasound images may in some cases be more difficult to interpret in the later stages of the operation than in the beginning of the operation. The aim of this paper is to explain the causes of surgically induced ultrasound artefacts and how they can be recognized and reduced.

Methods The theoretical reasons for artefacts are addressed and the impact of surgery is discussed. Different setups for ultrasound acquisition and different acoustic coupling fluids to fill up the resection cavity are evaluated with respect to improved image quality.
\end{abstract}

T. Selbekk $(\bowtie) \cdot$ F. Lindseth $\cdot$ I. Reinertsen

Department of Medical Technology, SINTEF,

PO Box 4760, Sluppen,

7465 Trondheim, Norway

e-mail: tormod.selbekk@sintef.no

T. Selbekk • A. S. Jakola • O. Solheim • T. F. Johansen •

F. Lindseth $\cdot$ G. Unsgård

Norwegian University of Science and Technology (NTNU),

Trondheim, Norway

A. S. Jakola $\cdot$ O. Solheim $\cdot$ G. Unsgård

St. Olavs Hospital, Trondheim University Hospital,

Trondheim, Norway

T. Selbekk $\cdot$ A. S. Jakola $\cdot$ O. Solheim $\cdot$ F. Lindseth

I. Reinertsen $\cdot$ G. Unsgård

National Competence Services for Ultrasound and Image-guided

Therapy, St. Olavs Hospital, Trondheim, Norway

I. Reinertsen

Medical Imaging Lab, NTNU, Trondheim, Norway

T. F. Johansen

Department of Acoustics, SINTEF, Trondheim, Norway
Results The enhancement artefact caused by differences in attenuation of the resection cavity fluid and the surrounding brain is the most dominating surgically induced ultrasound artefact. The influence of the artefact may be reduced by inserting ultrasound probes with small footprint into the resection cavity for a close-up view of the areas with suspected tumor remnants. A novel acoustic coupling fluid developed for use during ultrasound imaging in brain tumor surgery has the potential to reduce surgically induced ultrasound artefacts to a minimum.

Conclusions Surgeons should be aware of artefacts in ultrasound images that may occur during brain tumor surgery. Techniques to identify and reduce image artefacts are useful and should be known to users of ultrasound in brain tumor surgery.

Keywords Ultrasound · Neurosurgery · Brain tumors · Resection control · Ultrasound artefacts · Enhancement artefact · Ultrasonography $\cdot$ Intraoperative imaging

\section{Introduction}

Ultrasound is utilized in many neurosurgical departments worldwide for imaging of tumors in brain surgery. The purpose of ultrasound imaging is to locate the tumor and anatomical structures, as well as to identify residual tumor during surgery. By using real-time ultrasound imaging or 3D ultrasound-based navigation the surgeon can potentially discover and remove more of the tumor tissue [1-3].

For diffuse infiltrating gliomas, resection grade is associated with survival $[4,5]$. Ultrasound has the potential to increase resection grades, and thereby increase survival [6]. Ideally, high image quality should be sustained throughout the whole operation in order to monitor the progress of 
tumor resection. However, the progress of surgery may also cause more noise and more inaccurate display of the brain anatomy in the ultrasound images.

The term artefact is in medical imaging used to describe any part of the image that does not accurately represent the anatomy of the subject being investigated, and it is well known that ultrasound is prone to several different types of artefacts [7]. When using ultrasound in brain tumor surgery, the presence of artefacts may interfere the surgeon's interpretation of the images. The artefacts could make the images toward the end of surgery difficult to interpret, and could lead to small remnants of tumor being left behind. On the other hand, the image artefacts may also lead to overly aggressive resections if it is falsely interpreted as tumor remnant. Thus, awareness of these potential pitfalls is essential for successful ultrasound-guided brain tumor surgery.

This paper addresses the theoretical reasons for ultrasound artefacts, how they are affected by surgery, and its practical implications with respect to image interpretation. Different approaches to reduce image artefacts in intraoperative ultrasound imaging of brain tumors are described, including the development of a novel acoustic coupling fluid.

\section{Theory}

\section{Generation of ultrasound images}

Sound in the human audible range has frequencies between approximately $20-20,000 \mathrm{~Hz}$. Ultrasound is defined as sound with frequencies above $20 \mathrm{kHz}$. In medical ultrasound imaging, the frequency range is $2-40 \mathrm{MHz}$, with the highest frequencies currently used in intravascular ultrasound (IVUS).

Ultrasound imaging is a pulse-echo technique. The generation of the ultrasound images is based upon transmission of a sound pulse and recording of echoed events that has been reflected from tissue boundaries or scattered from smaller tissue structures. In conventional scanners today, a narrow ultrasound beam is transmitted from the ultrasound transducer. When the transmitted ultrasound pulse encounters objects with different acoustic properties, some of the energy of the transmitted sound pulse is echoed back to the transducer. After processing and depth conversion, the echoes received by the transducer are displayed as brightness versus depth on the ultrasound monitor, so-called B-mode imaging. The strength of the echo determines the brightness of the echo displayed in ultrasound images.

The echoes of the transmitted pulses are caused by differences in the mass density and compressibility of the medium that is explored by the ultrasound transducer.
When a transmitted ultrasound pulse meets boundaries between tissues or structures, for example between normal brain tissue and a tumor, the pulse is partially reflected backward and partially transmitted forward from the interface between the tissues. Smaller particles in the tissue may cause scattering of sound, which will also cause acoustic energy to be echoed back to the transducer. The ultrasound images will therefore consist of echoes originating from tissue interfaces, and echoes being scattered from particles/cells within the tissues. The strength of the echo is related to the differences in acoustic properties of the medium: The greater the difference, the stronger the echo.

Most brain tumors have higher mass density and sound velocity than the surrounding normal brain. In addition, the high cell density within the tumor will cause scattering of sound. A tumor will therefore usually appear in the ultrasound images as brighter than the surrounding normal brain, i.e., hyperechoic. Fluid-filled spaces like cysts and ventricles on the other hand will usually be seen in the ultrasound images as darker than the normal brain parenchyma, i.e., hypoechoic. This is because the fluid is usually very acoustically homogenous with few cells causing scattering of sound.

Propagation of sound in brain tissue

The ultrasound pulse emitted from the transducer array will propagate with a media dependent speed of sound, and the energy of the propagating pulse will decay with increasing distance from the transducer surface.

The speed of sound in air is approximately $330 \mathrm{~m} / \mathrm{s}$ and in fresh water approximately $1,480 \mathrm{~m} / \mathrm{s}$. In biological tissue, the speed of sound varies from approximately $600 \mathrm{~m} / \mathrm{s}$ in lung tissue to circa $4,000 \mathrm{~m} / \mathrm{s}$ in bone. In brain, the speed of sound has been measured to about $1,550 \mathrm{~m} / \mathrm{s}$ [8]. Ultrasound scanners made for medical imaging purposes assume a constant average speed of sound for the beam forming and the depth conversion. For imaging of soft biological tissue, the speed of sound is usually set to a value around $1,540 \mathrm{~m} / \mathrm{s}$. However, the value of the constant speed of sound may in some scanners vary with the user selected imaging application, to adjust for the expected speed of sound in the organ to be imaged.

The amplitude of the ultrasound pulse will gradually decrease as it propagates in the tissue. The attenuation is caused by conversion of the acoustic energy to heat and scattering or reflection of the ultrasound pulse out of the desired direction of propagation. The attenuation of a given medium is given by the attenuation coefficient $\alpha$, which usually is expressed as a damping value in decibel per megahertz per centimeter $[\mathrm{dB} /(\mathrm{MHz} \cdot \mathrm{cm})]$. Thus, the attenuation of the ultrasound pulse is heavily dependent on the attenuation coefficient of the investigated medium, the 
distance traveled and the frequencies of the pulse. Higher frequencies are more attenuated than lower frequencies. For soft biological tissue, a value of $\alpha=0.5 \mathrm{~dB} /(\mathrm{MHz} \cdot \mathrm{cm})$ is often used. However, measurements of attenuation in brain tissue suggest a higher value for the attenuation coefficient.

Bamber et al. reported measurements of attenuation in fresh human brain tissue acquired from autopsy [9]. They found that the attenuation in predominately white matter was higher than the attenuation in mixed grey and white matter with a ratio of 1.4. The attenuation, reported in $\mathrm{dB} / \mathrm{cm}$, ranged from 0.58 in mixed grey and white matter to 0.8 in tissue containing mostly white matter.

Kremkau et al. measured the attenuation and speed of sound in fresh tissue from five different anatomical locations of the brain provided by autopsy of four adults [10]. The average attenuation measured at a frequency of $1 \mathrm{MHz}$ was $0.87 \mathrm{~dB} / \mathrm{cm}$. The attenuation was found to be higher in white matter than in mixed tissue (white and gray matter) and in grey matter, with attenuation coefficients of approx. $0.97,0.87$, and $0.75 \mathrm{~dB} / \mathrm{cm}$, respectively.

Strowitzki et al. measured the attenuation of brain tissue in vivo from spectral analysis of ultrasound radiofrequency data acquired in 20 brain operations [11]. The slope of attenuation in the subcortical tissue with white matter dominance was found to be $0.94 \pm 0.13 \mathrm{~dB} /(\mathrm{MHz} \cdot \mathrm{cm})$.

The large attenuation coefficient for brain tissue found in the above-mentioned studies suggests that the ultrasound pulses will be more damped in the brain than in most other soft tissues.

Reasons for ultrasound artefacts in intraoperative imaging

The process of acquisition and generation of ultrasound images may itself introduce noise and distorted representations of the true anatomy in the ultrasound images. This noise and distortion seen in the images is referred to as ultrasound artefacts. The reason for the imperfect ultrasound image may be addressed to the violation of one or more of the following assumptions:

1. The ultrasound beam is narrow with uniform width

2. The ultrasound waves travel in a straight line directly to the reflecting object and back to the transducer

3. The speed of sound is constant and known in soft tissue

4. The attenuation of ultrasound is constant and uniform

The violation of the assumptions may become more pronounced as surgery progresses. Thus, the surgical resection of tumor may result in increased image artefacts and more challenges related to the interpretation of the ultrasound images.

Assumption No. 1 is violated by the fact that the ultrasound beam in reality is not infinitely thin, but is having a spatial extent that can be described by the axial, lateral and elevation resolution. The violation of the assumption can be seen in images as smearing of objects that are smaller than the actual beam width. The beam profile is mainly determined by the specifications of the ultrasound transducer array, and the spatial extent of the ultrasound beam should not be much affected by surgery.

Violation of assumption No. 2 can cause reverberations and mirror image artefacts. Neither of these two artefacts can be said to pose a significant problem in intraoperative imaging of brain tumors, but reverberations may in some cases be observed. Reverberations are caused by the ultrasound pulse being reflected multiple times, i.e., that the echoed sound received by the transducer has been reflected more than once. Reverberations can be seen as multiple reflections of the bottom of resection cavity, thus the artefact may become more apparent during surgery. This may or may not be considered to degrade the image quality, being dependent on the localization of the multiple reflections in the images. If the reverberations appear deeper in the images than the deepest target of interest, the added noise will probably not significantly degrade the clinical usefulness of the images. However, if reverberating events are appearing in the same depth and location as the primary reflection of a target of interest, this may be considered to degrade the ultrasound image quality. However, violation of assumption No. 2 alone should rarely generate artefacts that may be mistaken as remaining tumor tissue.

Assumption No. 3, that the speed of sound is constant and known in soft tissue, is more or less always violated in medical ultrasound imaging. This may cause improper delineation of geometry, depth range errors, and phase aberration. The phase aberration refers to the defocusing of the ultrasound beam, which is caused by distortions of the ultrasound wavefront due to differences in the speed of sound. Portions of the propagating wavefront will be advanced or retarded depending on the speed of sound, and this may cause distortions in the focusing and steering of the ultrasound beam. This may in turn lead to reduced resolution and contrast in the ultrasound images. Violation of assumption 3 is not a major issue when it comes to intraoperative ultrasound imaging of the brain, as the brain is a quite homogenous organ. The resection of tumor may potentially lead to some more depth inaccuracies and noise in the images due to the violation of the constant speed of sound assumption, as the saline water used for filling up the resection cavity will have a sound velocity of about $1,525 \mathrm{~m} / \mathrm{s}$ compared to about $1,550 \mathrm{~m} / \mathrm{s}$ that has been stated for brain tissue [8]. For a target at $10-\mathrm{cm}$ depth, this difference in velocity will introduce a depth range error of approximately $1.6 \mathrm{~mm}$.

Among the four assumptions listed, the assumption that the attenuation of tissue is constant and uniform (assumption No. 4 ) is the one most severely violated by surgery. The attenuation coefficient $\alpha$ may vary between different substances. For brain tissues, it has been measured to be in the range from approximately 0.65 to $0.95 \mathrm{~dB} /(\mathrm{MHz} \cdot \mathrm{cm})$. Apart from the 
obvious loss of frequency content and thereby resolution with depth, the attenuation of the brain does not induce any major ultrasound artefacts in the ultrasound imaging performed prior to resection. The time-gain-control (TGC) of the scanner can be adjusted to compensate for the attenuation of the ultrasound waves with distance. Thus, the images of the brain acquired prior to resection often have a homogenous appearance and high image quality.

After the start of resection, the presence of a resection cavity will introduce significant alternations in the attenuation of the imaged medium. The resection cavity needs to be filled with a saline solution, which practically has close to zero attenuation. The very large difference in attenuation between brain and saline might have a large negative impact on ultrasound images of the operation area. The difference in attenuation is causing the enhancement (or brightness) artefact, which is the most common and dominant image artefact in intraoperative ultrasound imaging of brain tumors. The artefact may be seen in the images as an increased brightness below the bottom of the saline filled cavity, which during brain surgery will be the resection cavity (Fig. 1).

If enhancement artefacts are present in the images, they may mask the presence of possible tumor remnants. It is therefore vital that the surgeon know how to identify this artefact, so it is not interpreted as remaining tumor tissue that may be resected.

\section{How to separate artefacts from true events}

Although the enhancement artefact may be profound when a large resection cavity is established, it is possible to determine if an enhanced signal is caused by predominately a highly echogenic tissue or the low attenuation of water relative to brain tissue. In real-time 2D imaging, the location of the artefact in the image will move when the probe is moved or altered in position and angle. There are also technical systems integrating ultrasound imaging and navigation technology for use in brain tumor surgery [12]. One of these systems permits the surgeon to acquire 3D

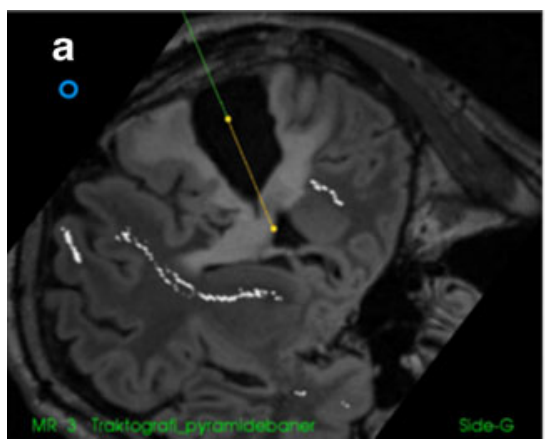

Fig. 1 A screen display from a 3D ultrasound-based navigation system (SonoWand Invite) showing a reformatted image slice from the preoperative MRI volume (a), and the same MR image slice with the ultrasound image volumes throughout surgery, and navigation of surgical instruments may be done based on intraoperative ultrasound images. The acquisition of a new 3D ultrasound volume is done very rapidly. Typically it takes less than a minute from start of ultrasound acquisition to the navigation can be performed. The surgeon should preferably acquire 3D ultrasound volumes multiple times during the tumor resection. Towards the end of the procedure, the most recently acquired image volume should be compared to the volumes acquired at an earlier stage of the surgery. If a high-intensity region can be observed at the same location in all image volumes, it is likely to be caused by highly echogenic tissue as, e.g., tumor tissue. If the signal enhancement can only be observed in the last acquisitions performed through the saline filled resection cavity, the signal enhancement is likely to be an artefact (Fig. 2).

\section{Approaches to minimize ultrasound artefacts}

When using ultrasound for resection control, it is important for safety of the procedure that image artefacts are recognized. However, it is possible to reduce the influence of surgically induced artefacts in the images.

As pointed out previously, the most pronounced ultrasound artefact that can be observed in intraoperative ultrasound imaging in brain tumor surgery is the enhancement artefact caused by differences in attenuation in the medium investigated. The damping of the ultrasound pulse is related to the attenuation coefficient $\alpha$ of the media and the distance traveled. The artefact can be minimized by either reducing the distance between the ultrasound transducer and the imaged region of interest, or by making the attenuation coefficient $\alpha$ equal in the whole imaged area.

Inserting probes in the resection cavity

During surgery the ultrasound probes are usually positioned at the brain cortex when ultrasound imaging is performed.

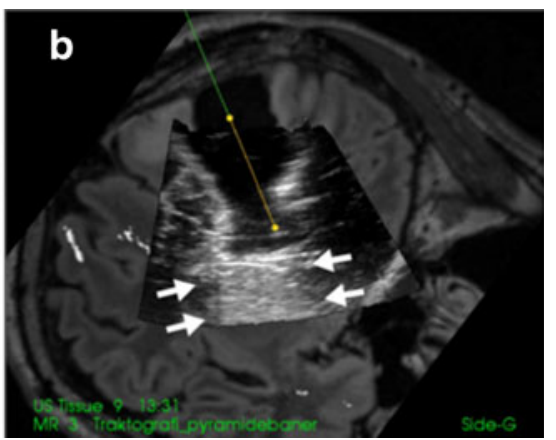

corresponding ultrasound image as overlay (b). The enhancement artefact below the bottom of the long resection cavity is marked with bright arrows in $\mathbf{b}$ 

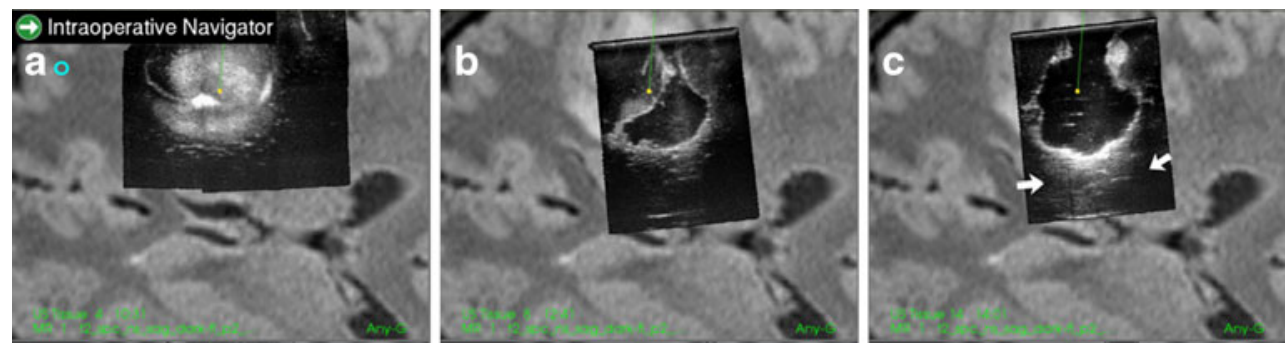

Fig. 2 Navigation display showing the reformatted ultrasound image slices on top of the corresponding reformatted MR image slice. Ultrasound image slice from 3D ultrasound volume acquired prior to start of resection (a), towards the end of resection with some tumor tissue

When a resection cavity has been established, it may in many cases be possible to insert probes of small size and footprint into the resection cavity (Fig. 3). In this way, the probe can be brought within close distance to the bottom of the resection cavity to inspect the tissue of the resection margins. This approach will reduce or remove the brightness artefacts, as the distance from the probe surface to the imaged object is shorter. A possible drawback is that the width of the ultrasound image is bound to be limited due to the small size of the ultrasound probe. Only smaller sections of the resection margin can be investigated at a time. This approach may be used to supplement imaging with conventional ultrasound probes if a small area of suspicion is detected. If 3D ultrasound acquisition is available, moving the probe back and forth over the area with possible tumor remnants may provide an ultrasound volume that covers the region of interest.

A novel fluid with acoustic properties mimicking brain tissue

When acquiring 3D ultrasound data during tumor resection, the probe may be placed partly over the resection cavity remaining (b), and after completed tumor resection (c). Notice the signal enhancement below the cavity (marked with arrows) seen in $\mathbf{c}$, which is not observed in a or $\mathbf{b}$. Hence, it is very likely that the enhancement is an artefact and not remaining tumor

filled with saline (or other physiologic solutions like, e.g., Ringer). To avoid the enhanced brightness below the resection cavity, the ultrasound pulses propagating in the saline solution would need to experience a similar attenuation as in the brain parenchyma.

Our cross-disciplinary research group within ultrasound and image-guided therapy in Trondheim, Norway, has recently developed a fluid with attenuation similar to the brain. A patent application has been submitted. The fluid has the potential to replace saline as an acoustic coupling medium for intraoperative ultrasound imaging. The fluid has an attenuation coefficient $\alpha$ targeted at $0.80 \mathrm{~dB} /(\mathrm{MHz} \cdot \mathrm{cm})$, which is in the same order as $\alpha$ of the adult human brain. Further, the fluid is sufficiently viscous to allow easy removal from the resection cavity with a suction device after imaging. The imaging properties of the fluid have been evaluated on phantoms in the laboratory (Fig. 4) and on fresh piglet cadavers (Fig. 5).

Assessment of potentially harmful effects of the fluid has been tested in animal studies where the fluid has been injected in brain parenchyma and cerebrospinal fluid. The results of the animal experiments have been submitted for publication.
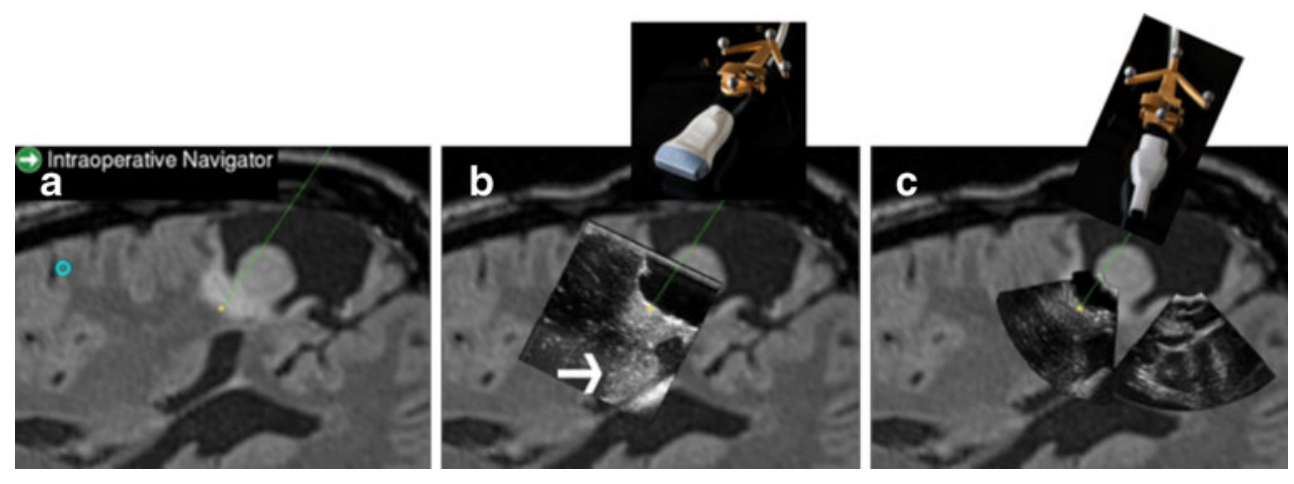

Fig. 3 Screen display from a 3D ultrasound-based navigation system (SonoWand Invite) showing the image slice of preoperative MR (a) that is reformatted according to the orientation and angle of the navigation instrument. The corresponding reformatted image slice from an ultrasound volume that was acquired with a flat linear array probe (shown above for illustration) positioned at the brain cortex is shown in b. The reformatted ultrasound image slice from an ultrasound volume acquired with a small-sized phased array probe (shown above for illustration) inserted in the resection cavity (c). In b and c, the ultrasound volumes are acquired after part of the tumor is removed. Notice enhancement artefact in b marked with a bright arrow, which is not present in $\mathbf{c}$ where the data has been acquired with the probe in close distance to the bottom of the cavity 


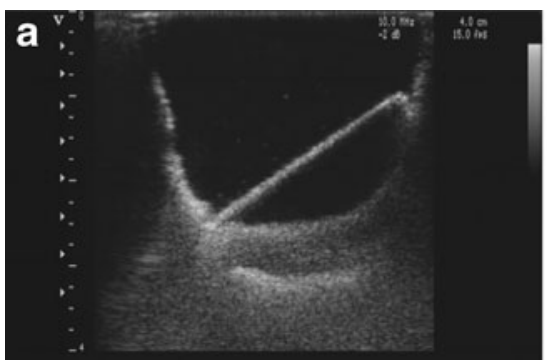

Fig. 4 A needle inserted in a cavity made in oasis, imaged by a flat linear array (Vingmed Ultrasound System FiVe) with regular saline shown to the left (a), and imaged with a fluid with higher attenuation shown to the right (b). The same acquisition parameters were used, but

Since the fluid is opaque, it will be difficult to use the fluid in combination with 2D ultrasound imaging for realtime monitoring of surgical instruments. To fully exploit the usefulness of the brain-mimicking acoustic coupling fluid, it should be used in combination with acquisition of 3D ultrasound volumes during surgery. We are currently awaiting approvals for the use in selected patients for a phase 1 feasibility study using intraoperative 3D ultrasound for guidance and resection control.

\section{Discussion}

In this paper, we present theoretical reasons for ultrasound artefacts and practical solutions to reduce the impact of artefacts when ultrasound is used for resection control in brain tumor surgery.

As discussed, ultrasound artefacts may limit overall image quality. When it comes to detecting tumor remnants, the enhancement artefact can be especially problematic, since a hyper-echoic zone around the cavity may both mask

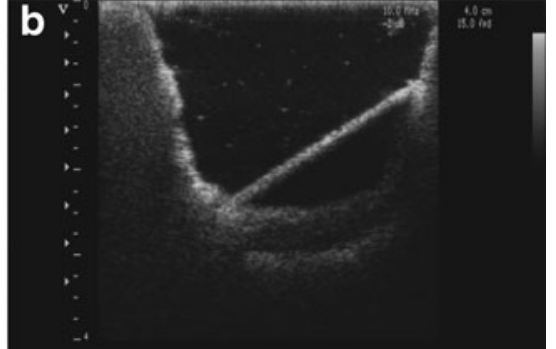

the global gain was adjusted in each case to provide the "best image" as defined by an experienced ultrasound operator. Note that the bright region below the bottom of the cavity in a is almost completely vanished in $\mathbf{b}$

significant tumor remnants and falsely resemble tumor remnants. The novel acoustic coupling fluid presented in the paper seems promising in reducing the influence of image artefacts, and would make image acquisition and image interpretation towards the end of surgery less dependent on a long learning curve.

Ultrasound is used for resection control in many neurosurgical units worldwide, but only a few studies have investigated the use of ultrasound for resection control in brain surgery. A recent study suggests that the use of $3 \mathrm{D}$ ultrasound in diffuse low-grade gliomas makes an aggressive attitude feasible, thereby improving survival without increase in morbidity [4].

Ultrasound image findings towards the end of surgery have been compared with histology from biopsies in a few studies. LeRoux et al. and Woydt et al. compared real-time 2D ultrasound images of gliomas with histopathology, and concluded that intraoperative US could improve gross total resection [13, 14]. Chacko et al. compared 2D ultrasound images at the end of tumor resection with histopathology and found that of the 79 samples taken from the tumor-brain interface that were reported as tumor on ultrasound, 66 had histopathological
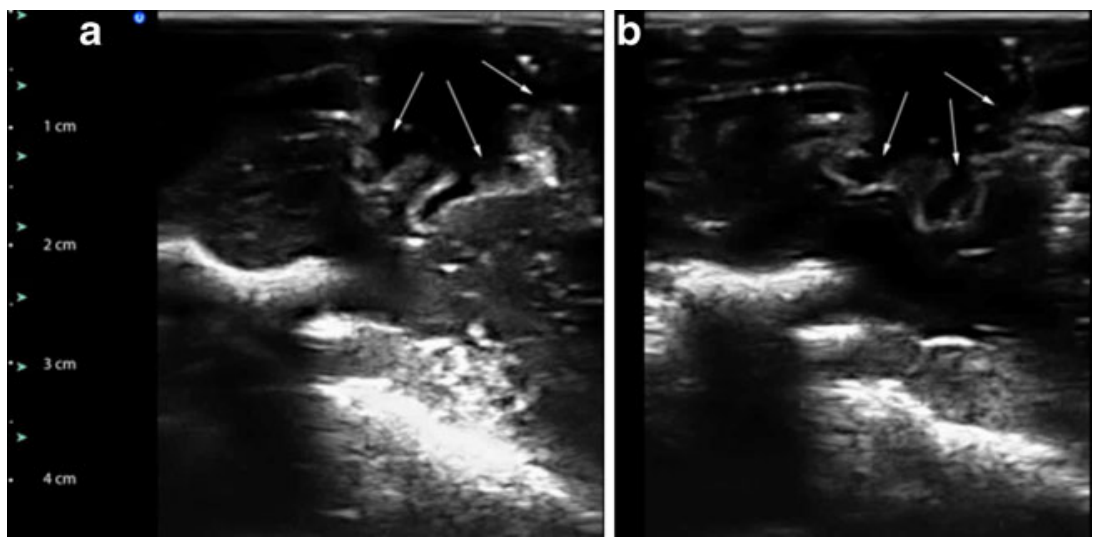

Fig. 5 Resection cavity (marked with arrows) made in a fresh piglet brain, imaged using physiological saline to fill the resection cavity (a) and imaged using the developed acoustic coupling fluid (b). The acquisition parameters are identical in both cases. The dominating signal enhancements in the deeper part of the images are caused by strong reflections from the scull base. Note the difference in intensity below the resection cavity in the two images. Even if the slice orientation is not perfectly identical in the images, it is apparent that there is no artificial signal enhancement directly below the bottom of the resection cavity in $\mathbf{b}$. The difference in signal intensity can also be seen in the deeper bone reflections in $\mathbf{a}$ and $\mathbf{b}$ 
evidence of tumor while 13 samples were negative for tumor, giving a positive predictive value of 0.84 [15].

Navigated 3D ultrasound has been used for image-guided biopsy sampling in three different stages of glioblastoma resection [16]. The results showed an excellent agreement between ultrasound findings and histology for 3D ultrasound images acquired prior to the start of tumor resection. For ultrasound volumes acquired during resection and after completed resection, the ultrasound image findings had a somewhat less favorable agreement with histology.

The importance of maintaining good ultrasound image quality throughout the operation was also illustrated in a retrospective study of patients with glial tumors operated with 3D ultrasound imaging [17]. The study found a significant relationship between resection grade and ultrasound image quality, i.e., good ultrasound image quality was found to be associated with higher resection grade.

In this paper, we have suggested some approaches to reduce the influence of surgically induced ultrasound image artefacts in resection of brain tumors.

So far, ultrasound images of brain tumors have mostly been acquired from the cortex of the brain, outside the lesions. With the miniaturization of probes, it is possible to make highresolution image acquisitions from within the lesions as well. The reduced distance from probe to the tissue of interest enables the use of higher frequencies and thereby obtaining images with higher resolution. In addition, a reduced distance between the ultrasound transducer and the resection-brain interface will reduce the vulnerability to enhancement artefacts, as also pointed out by Steno et al. [18]. Such specially designed cavity probes may be tightly curved, high-frequency, linear array probes that may be placed directly on the tissue in areas where the surgeon is uncertain about the extent of resection, e.g., in regions with enhancements seen in $3 \mathrm{D}$ image volumes. In addition to reducing the enhancement artefacts, such small probes may also record images without removal of spatulas. This reduces brain shift and increases accuracy.

Geirmund Unsgård et al. have advocated the importance of proper patient positioning and optimizing of the ultrasound acquisition setup [3]. They suggested that ultrasound acquisition should be made on intact dura if possible, in a slightly enlarged craniotomy. A separate mini-craniotomy for the ultrasound transducer, with approximately a $90^{\circ}$ angle to the operating channel, could also be made to acquire high-quality ultrasound images. The latter approach has not been used at our institution in the last decade. However, both approaches will likely reduce the influence of the enhancements artefact, as the imaging directly through the fluid filled operating channel is avoided. If space permits, the imaging could be done with the transducer held on intact dura instead of placing the transducer on top of the operating channel. This approach may be feasible for ultrasound transducers having a small footprint, as phased array probes.
A coupling fluid that attenuates ultrasound energy like the normal brain may perhaps be a more flexible solution to reduce the enhancement artefacts. By filling the resection cavity with the fluid before image acquisition, one could ensure that any hyper-echoic region seen adjacent to the cavity represent remaining brain tumor tissue and not artefacts. In addition to facilitating the interpretation of images, the avoidance of these common artefacts is expected to improve the efficacy of intraoperative ultrasound.

\section{Conclusions}

Awareness of artefacts in ultrasound images that may occur during brain tumor surgery is a necessity for successful and safe surgery when using ultrasound imaging for resection control. All users of ultrasound in brain tumor surgery should be familiar with techniques on how to identify and reduce image artefacts, as described in this paper. A novel acoustic coupling fluid has the potential to minimize artefacts during intraoperative ultrasound imaging, and may represent a valuable future tool for optimizing the riskbenefit ratio in brain tumor surgery.

Acknowledgments The development of the brain-mimicking acoustic coupling fluid has been financed by grants from the Ministry of Health and Care Services, through the National Competence Services for Ultrasound and Image-guided Therapy, at St. Olavs University Hospital, Trondheim, Norway.

Disclosure GU has $0.1 \%$ of the shares in the company SonoWand AS (Trondheim, Norway), and may indirectly benefit economically from increased use of 3D ultrasound in neurosurgery. GU, TS, and TFJ may benefit from possible future of acoustic coupling fluid, as inventors in the pending patent.

\section{Conflicts of interest None.}

Open Access This article is distributed under the terms of the Creative Commons Attribution License which permits any use, distribution, and reproduction in any medium, provided the original author(s) and the source are credited.

\section{References}

1. Unsgaard G, Ommedal S, Muller T, Gronningsaeter A, Nagelhus Hernes TA (2002) Neuronavigation by intraoperative threedimensional ultrasound: initial experience during brain tumor resection. Neurosurgery 50:804-812, discussion 812

2. Unsgaard G, Rygh OM, Selbekk T, Muller TB, Kolstad F, Lindseth F, Hernes TA (2006) Intra-operative 3D ultrasound in neurosurgery. Acta Neurochir (Wien) 148:235-253, discussion 253

3. Unsgård G, Gronningsaeter A, Ommedal S, Nagelhus Hernes TA (2002) Brain operations guided by real-time two-dimensional ultrasound: new possibilities as a result of improved image quality. Neurosurgery 51:402-412 
4. Jakola AS, Myrmel KS, Kloster R, Torp SH, Lindal S, Unsgård G, Solheim O (2012) Comparison of a strategy favoring early surgical resection vs a strategy favoring watchful waiting in low-grade gliomas. JAMA J Am Med Assoc 308:1881-1888

5. Stummer W, Reulen HJ, Meinel T, Pichlmeier U, Schumacher W, Tonn JC, Rohde V, Oppel F, Turowski B, Woiciechowsky C, Franz K, Pietsch T (2008) Extent of resection and survival in glioblastoma multiforme: identification of and adjustment for bias. Neurosurgery 62:564-576, discussion 564-576

6. Sæter CA, Torsteinsen M, Torp SH, Sundstrøm S, Unsgård G, Solheim O (2012) Did survival improve after the implementation of intraoperative neuronavigation and 3D ultrasound in glioblastoma surgery? A retrospective analysis of 192 primary operations. J Neurol Surg A Cent Eur Neurosurg 73:73-78

7. Feldman MK, Katyal S, Blackwood MS (2009) US artifacts. Radiogr Rev Publ Radiol Soc North Am Inc 29:1179-1189

8. Duck FA (1990) Physical properties of tissue. Academic Press, London

9. Bamber JC (1981) Ultrasonic attenuation in fresh human tissues. Ultrasonics 19:187-188

10. Kremkau FW, Barnes RW, McGraw CP (1981) Ultrasonic attenuation and propagation speed in normal human brain. J Acoust Soc Am 70:29-38

11. Strowitzki M, Brand S, Jenderka KV (2007) Ultrasonic radiofrequency spectrum analysis of normal brain tissue. Ultrasound Med Biol 33:522-529

12. Grønningsæter $\AA$, Kleven A, Ommedal S, Årseth TE, Lie T, Lindseth F, Langø T, Unsgård G (2000) SonoWand, an ultrasound-based neuronavigation system. Neurosurgery 47:13731380

13. Le Roux PD, Berger MS, Wang K, Mack LA, Ojemann GA (1992) Low grade gliomas: comparison of intraoperative ultrasound characteristics with preoperative imaging studies. J Neurooncol 13:189-198

14. Woydt M, Krone A, Becker G, Schmidt K, Roggendorf W, Roosen K (1996) Correlation of intra-operative ultrasound with histopathologic findings after tumour resection in supratentorial gliomasA method to improve gross total tumour resection. Acta Neurochir (Wien) 138:1391-1398

15. Chacko AG, Kumar NK, Chacko G, Athyal R, Rajshekhar V (2003) Intraoperative ultrasound in determining the extent of resection of parenchymal brain tumours - a comparative study with computed tomography and histopathology. Acta Neurochir (Wien) 145:743-748, discussion 748

16. Rygh OM, Selbekk T, Torp SH, Lydersen S, Hernes TA, Unsgaard $\mathrm{G}$ (2008) Comparison of navigated 3D ultrasound findings with histopathology in subsequent phases of glioblastoma resection. Acta Neurochir (Wien) 150:1033-1041, discussion 1042

17. Solheim O, Selbekk T, Jakola AS, Unsgård G (2010) Ultrasoundguided operations in unselected high-grade gliomas-overall results, impact of image quality and patient selection. Acta Neurochir (Wien) 152:1873-1886

18. Steno A, Karlik M, Mendel P, Cik M, Steno J (2012) Navigated three-dimensional intraoperative ultrasound-guided awake resection of low-grade glioma partially infiltrating optic radiation. Acta Neurochir (Wien) 154:1255-1262 